\title{
Implicit associative learning in synesthetes and nonsynesthetes
}

\author{
Kaitlyn R. Bankieris ${ }^{1} \cdot$ Richard N. Aslin ${ }^{1}$
}

Published online: 9 September 2016

(C) Psychonomic Society, Inc. 2016

\begin{abstract}
Although cross-modal neural connections and genetic underpinnings are prominent in most current theories regarding the development of synesthesia, the potential role of associative learning in the formation of synesthetic associations has recently been revitalized. In this study, we investigated implicit associative learning in synesthetes and nonsynesthetes by recording reaction times to a target whose color was probabilistically correlated with its shape. A continuous measure of target detection at multiple time points during learning revealed that synesthetes and nonsynesthetes learn associations differently. Specifically, our results demonstrated a "fast-facilitation" learning effect for nonsynesthetes and a "fastinterference, slow-facilitation" learning effect for synesthetes. Additionally, synesthetes exhibited superior long-term memory for the learned associations in a surprise delayed retest. After this retest, participants implicitly learned new (shuffled) shape-color associations. We found that synesthetes experienced greater interference while learning these new shape-color associations. These results detail ways in which implicit associative learning and memory differ between synesthetes and nonsynesthetes.
\end{abstract}

Keywords Human associative learning - Implicit learning and memory . Synesthesia

Kaitlyn R. Bankieris

kbankier@ur.rochester.edu

1 Department of Brain and Cognitive Sciences, University of Rochester, Rochester, NY, USA
Synesthetes automatically and consistently experience additional, unusual cognitive or sensory experiences in response to particular environmental stimuli. For example, graphemecolor synesthetes experience specific colors for individual letters and numbers (i.e., 5 is magenta). These automatic, conscious, stable, and precise associations are the hallmark of synesthesia and have been documented for over a century, but their formation remains largely undetermined. The majority of current synesthesia theories focus on its genetic underpinnings and on cross-modal neural connections as the basis for synesthesia (e.g., Asher et al., 2009; Grossenbacher \& Lovelace, 2001; Ramachandran \& Hubbard, 2001), but early theories considered associative learning to be the causal mechanism of synesthetic experiences (Calkins, 1893; Claparede, 1903). Recent evidence has revitalized interest in the potential role of associative learning in the development of synesthesia. Moreover, these approaches need not be incompatible with one another, since a propensity to develop synesthesia may be genetic, whereas one's particular synesthetic experiences may be influenced by learning.

Recent evidence has suggested that a synesthete's associations do arise in part due to learning, highlighting the possibility that learning as a general cognitive function may differ in synesthetes. Since synesthesia is neither taught nor intentionally acquired by children, implicit learning in particular is potentially altered in synesthetes. Witthoft and Winawer (2013) reported that in a sample of 11 grapheme-color synesthetes, grapheme-color pairings largely overlapped with the colors of toy letters and numbers to which the synesthetes had been exposed as children. This finding suggests that the grapheme-color pairings the synesthetes experienced as young children influenced the formation of their synesthetic associations. This same research group later conducted a large-scale study investigating the influence of such colored toys on 6,588 grapheme-color synesthetes' associations 
(Witthoft, Winawer, \& Eagleman, 2015). They found that approximately $6 \%$ of synesthetes had a large number of synesthetic associations (10-26 per individual) that had been influenced by toys with colored graphemes. The researchers made this retrospective inference by charting the years preceding the introduction of a specific colored toy set to the commercial market, and finding that no participants' synesthetic experiences had a large overlap with the colored toys. In the decade following the availability of this toy set to consumers, however, nearly $15 \%$ of participants exhibited significant overlap between their synesthetic experiences and the toy set. These findings suggest that exposure to a particular set of environmental stimuli can influence the formation of synesthetic associations, suggesting that associative learning in early childhood plays a role in synesthesia.

Additional findings on the patterns of synesthetic experiences support the role of implicit learning in synesthesia. Although synesthetic experiences are superficially idiosyncratic from one synesthete to the next (e.g., the month November might be purple for one synesthete but orange for another), patterns emerge when looking at populations of synesthetes. For instance, sound-color synesthetes tend to associate higher pitches with lighter colors (Ward, Huckstep, \& Tsakanikos, 2006). Moreover, these patterns found in synesthetes' explicit associations match intuitions found in the general population of nonsynesthetes, as revealed by forced-choice cross-sensory association tasks (e.g., Bankieris \& Simner, 2014; Cytowic \& Wood, 1982; Marks, 1974, 1987; Simner et al., 2005; Simner \& Ludwig, 2012; Smilek, Carriere, Dixon, \& Merikle, 2007). Although some of these commonalities may arise due to magnitude comparisons across senses, others - such as those including linguistic components-are necessarily learned. These common patterns across synesthetes and nonsynesthetes suggest that synesthetic associations may arise in part due to a sensitivity to associative pairings or environmental statisticsparticularly early in development.

Despite the potential role of implicit learning in synesthesia, only one study to date has investigated differences in implicit-learning abilities between synesthetes and nonsynesthetes. In this artificial-grammar learning study, grapheme-color synesthetes, sequence-space synesthetes, and nonsynesthetes were exposed to two different grammars within the training phase of the experiment - one with symbols and one with letters (Rothen et al., 2013). Strings were presented one character at a time, and participants wrote down the entire string at the end of its presentation. After the training phase, participants were informed that the strings had been generated by a set of rules. At test, participants made grammaticality judgments on strings from the grammars they had been exposed to during learning, in addition to two novel grammars. Grapheme-color synesthetes performed more accurately than controls on letter strings but not symbol strings, suggesting that their increased accuracy was related to their synesthetic color experiences triggered by the letter strings. Supporting this interpretation of the results, sequence-space synesthetes - whose synesthesia occurs in a domain outside of the materials used in this study - did not perform more accurately than controls on either grammar. Accordingly, this study does not support the hypothesis that synesthetes have extraordinary implicit-learning abilities, but rather exhibit superior learning because of the benefit provided by their "extra" synesthetic experience.

In the present study, we specifically investigated implicit associative learning (rather than grammar, rule, or sequence learning) in synesthetes and nonsynesthetes. Mimicking the natural environment in which synesthesia develops, we presented associations in a probabilistic manner and did not require participants to attend to these associations to perform the experimental task. We collected a continuous dependent measure (reaction time, RT) on each trial to estimate a smooth learning curve, and repeated testing across days and weeks to investigate potential long-term memory differences between synesthetes and nonsynesthetes. With this probabilistic design, we not only analyzed the RTs to "correct" associations, but also evaluated the RTs to "incorrect" associations as a way to investigate any interference experienced for improbable shape-color pairings. Finally, since synesthetes' associations generally do not change abruptly, we introduced novel associations (same shapes, different colors) during the final session to assess potential group differences in the ability to implicitly learn new associations. We sought to investigate synesthetes' abilities to implicitly learn, retain, and relearn shape-color associations with this multipart experimental design. If synesthetes have a general superior implicit associative-learning ability, we should see that synesthetes learn these probabilistic associations more quickly than controls. If this hypothesized associative learning ability comes at the cost of stronger interference for incorrect associations, we would expect synesthetes to be more affected by the presentation of incorrect associations and to be worse at relearning shuffled shape-color associations than controls would be.

\section{Method}

\section{Participants}

Eight linguistic-color synesthetes, who experienced colors for letters, numbers, days of the week, and/or months of the year, were recruited from our preexisting database of Rochesterarea synesthetes. Seven of the synesthetes completed all portions of the study, and we recruited nonsynesthetes from the Rochester area until we had seven participants approximately matched for age and education who had also completed all portions of the study (see Table 1 for the participant information). This resulted in the exclusion of four nonsynesthetes 
Table 1 Participant information

\begin{tabular}{lllllll}
\hline \multicolumn{2}{l}{ Synesthetes } & & & \multicolumn{2}{l}{ Nonsynesthetes } \\
\cline { 1 - 1 } \cline { 6 - 7 } Age & Gender & Education & & Age & Gender & Education \\
\hline 18 & F & HS & & 18 & M & HS \\
18 & F & HS & & 20 & M & HS \\
19 & F & HS & & 21 & M & HS \\
20 & F & HS & & 22 & F & HS \\
26 & F & MS & & 27 & F & BS \\
27 & F & MS & & 28 & M & MS \\
42 & M & MS & & 41 & M & MS \\
\hline
\end{tabular}

"Education" indicates the highest level completed

and one synesthete who did not complete all portions of the study. All participants were compensated $\$ 10 / \mathrm{h}$ for their participation. Ethical approval was obtained from the University of Rochester Research Subjects Review Board.

Our synesthetes' self-reported experiences had previously been confirmed with an objective test of genuineness - consistency over time - presented via the diagnostic website http://synesthete.org (see Eagleman, Kagan, Nelson, Sagaram, \& Sarma, 2007, for the methods). This test identifies synesthetes on the basis of replicated findings that synesthetes are significantly more consistent when repeatedly choosing synesthetic colors for the stimuli eliciting them (e.g., letters) than are nonsynesthetes. Our synesthetes experienced colors in response to letters and/or numbers $(n=4)$, days of the week $(n=4)$, and/or months of the year $(n=3)$, as confirmed by mean standardized scores of $0.66(S D=0.28), 0.63(S D=0.13)$, and $0.50(S D=0.09)$, respectively, where a score below 1 confirms synesthesia (see Eagleman et al., 2007, for details). Two synesthetes experienced colors for graphemes, days of the week, and months of the year; two experienced colors in response to days of the week and months of the year; one experienced colors only for days of the week; and two experienced colors only for graphemes. Nonsynesthetes completed a synesthesia questionnaire (see http://synesthete.org) and indicated no synesthetic experiences.

\section{Stimuli}

The stimuli consisted of ten discriminable "snowflake" shapes created with ART-TEK's online snowflake generator (link inactive at time of publication: www.art-tek-ltd.co. uk/snowflake/; please contact the corresponding author for the stimuli). During the implicit-learning task, these snowflakes were presented in six canonical colors: red, blue, green, cyan, magenta, and yellow.

\section{Procedure}

Participants implicitly learned two sets of snowflake-color pairings across two in-lab "visits": The first visit spanned two days, and the second visit occurred on one day at least two weeks after the first visit. At the beginning of the first day, three snowflakes were randomly selected as targets for the detection task, with the remaining seven snowflakes used as distractors. Participants first completed a pretest during which they were shown six snowflakes (the three target snowflakes and three randomly selected distractors), rendered in white on a gray background, one at a time and asked whether they consistently and automatically associated each snowflake with a color. If participants indicated that they did have an associated color for the presented snowflake, participants chose their associated color from a color wheel.

Following the pretest, participants completed six blocks of 135 visual detection trials modeled after Kusnir and Thut (2012). Each trial began by displaying one of the three target snowflakes in black in the center of the screen for $1 \mathrm{~s}$, as can be seen in Fig. 1a. The target snowflake was then replaced by six colored snowflakes: the target snowflake and five distractor snowflakes. The array of six colored snowflakes remained on the screen until participants indicated whether the target snowflake was presented on the left or the right side of the screen, by pressing the appropriate arrow key. Unbeknownst to the participants, across trials two of the target

\section{a}

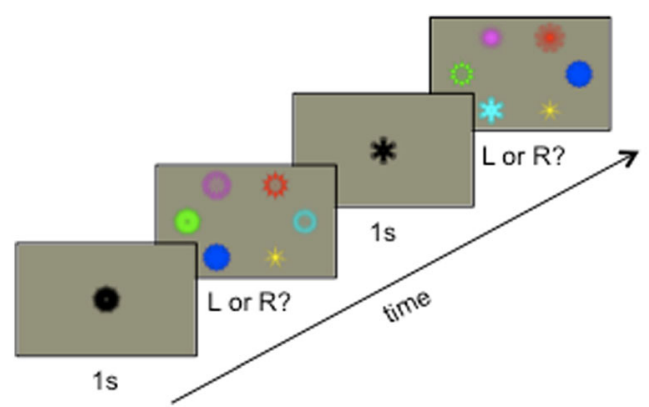

b
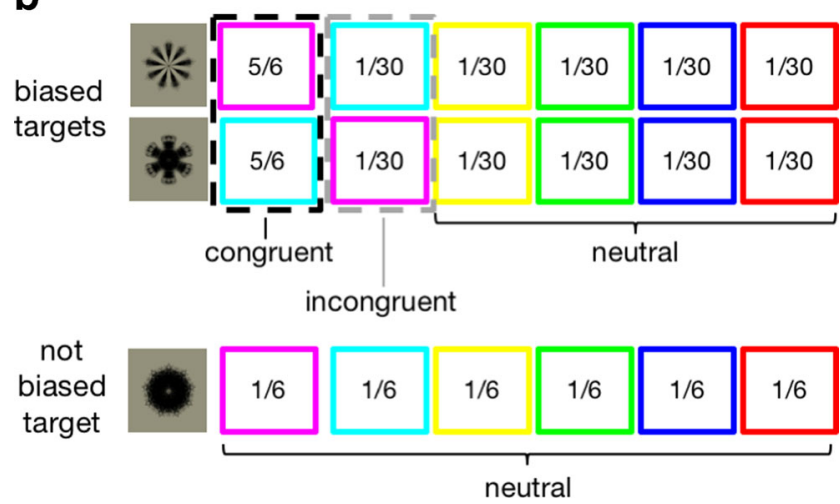

Fig. 1 Experimental procedure and stimuli. (a) Example of detection trials in which participants indicate whether the target snowflake (black) appears on the left or the right side of the screen. (b) Probabilities of target snowflakes appearing in each of the six colors 
snowflakes were not presented in the six colors equally (i.e., they were color-biased, as is shown in Fig. 1b). The colorbiased targets were presented in their "congruent" color $83.3 \%(5 / 6)$ of the time, and presented in every other color $3.3 \%$ of the time (i.e., $1 / 6 * 1 / 5=1 / 30$ ). The remaining target was unbiased in color presentation, appearing in each color $16.7 \%(1 / 6)$ of the time. Thus, the three target snowflakes were equiprobable across trials, but for two of the target snowflakes their assigned colors in the six-snowflake search array were not equiprobable.

After completing six visual-detection blocks with a 1-min break after each, participants reported their associations by completing a task similar to the pretest. Again they were shown six white snowflakes one at a time (the three target snowflakes and three randomly selected distractors), but they were now asked whether they had begun to associate a color with that snowflake based on their experience in the previous sections. If participants responded "yes," they selected the associated color from a color wheel and submitted their answer. Additionally, the participants reporting an association were next shown the six colors used in the experiment and asked to click on their associated color if it was present, or to press " $n$ " if they did not associate the snowflake with one of the six colors presented. We will refer to this task as the association report task, hereafter.

Participants returned to the lab the following day to complete their first visit. The second day consisted of the association report task, six blocks of detection trials, a repetition of the association report task, and a computerized version of the Corsi block-tapping task, to assess working memory (Mueller, 2011).

At least two weeks after the initial visit, participants returned for a surprise second visit (seven synesthetes, seven controls). This second visit began with the association report task, asking participants to recall the snowflake-color pairings they had learned two weeks previously. Participants then completed six blocks of detection trials. In this session, however, the congruent colors for the biased targets were changed (e.g., a target snowflake previously presented in magenta five-sixths of the time was now presented in blue five-sixths of the time). The experiment concluded with the association report task.

\section{Data analysis}

We fit the detection task data with a set of linear mixed-effects regression models. We modeled the RTs of correct trials, which presented color-biased and non-color-biased target stimuli in congruent colors and neutral colors, excluding trials on which the color-biased target stimuli were presented in incongruent colors, due to their inherent infrequency. To control for general task learning unrelated to learning of the particular shape-color associations, we compared RTs to the color-biased targets to those of the non-color-biased snowflake. Formally, we created a factor termed Target Type with three levels: (1) non-color-biased target (necessarily presented in a neutral color), (2) color-biased target presented in its congruent color, and (3) color-biased target presented in a neutral color. In the Results section below, we present two regressions evaluating RT behavior during the first visit (across two successive days); one model compares congruent color-biased targets to the non-color-biased targets, and the other compares neutral color-biased targets to the non-color-biased targets. We refer to these models as the Visit 1 congruent and Visit 1 neutral models, respectively. Separating these comparisons allowed us to decrease collinearity (by sum-coding our factors) while maintaining meaningful, interpretable coefficients (because each factor now had only two levels). Each model fit $\log$ RTs with a number of predictors: $\log$ visit block, group, target type, and all interactions, as well as random intercepts and slopes by participant. We additionally report the results from a model comparing congruent color-biased trials to the non-color-biased trials from both visits, with a regression termed both visits congruent. This model additionally included visit and all of its interactions as fixed effects as well as an additional random effect (see Tables 2, 3, and 4 for the specific model details).

\section{Results}

In this study, we presented synesthetes and nonsynesthetes with snowflake-color associations embedded in a detection task. Before comparing the synesthetes' and nonsynesthetes' performance, we asked whether having a synesthetic color association with any of the target snowflake shapes affected performance on the implicit-learning tasks. We found no differences between the stimulus groups (preexisting snowflakecolor $[n=13]$; no synesthetic association $[n=28]$ ), and present the following analyses with these groups collapsed.

We conducted a series of regression analyses to answer four main questions regarding implicit associative learning in synesthetes and nonsynesthetes.

1. Do synesthetes learn initial associations more quickly than do controls? If synesthetes have a general implicit associative-learning advantage, their RTs to color-biased targets presented in congruent colors should decrease more quickly than controls' (controlling for each group's RT to the non-color-biased targets ${ }^{1}$ ).

2. Do synesthetes experience greater interference for "incorrect" associations? We would expect to see slower RTs to color-biased snowflakes presented in neutral colors

\footnotetext{
${ }^{1}$ For the rest of this article, all discussion of color-biased target RTs will be based on controls for non-color-biased RTs, but we refrain from explicitly stating this every time to avoid lengthy and confusing sentences.
} 
Table 2 Visit 1 congruent model fixed-effect results

\begin{tabular}{lrrr}
\hline & \multicolumn{3}{l}{$\beta$} \\
\hline Intercept & 6.42 & $(-0.05)$ & $* * *$ \\
log Visit Block & -0.16 & $(0.01)$ & $* * *$ \\
Group & -0.01 & $(0.05)$ & \\
Target Type & -0.10 & $(0.02)$ & $* * *$ \\
log Visit Block $\times$ Group & 0.02 & $(0.01)$ & \\
log Visit Block $\times$ Target Type & -0.01 & $(0.01)$ & \\
Group $\times$ Target Type & 0.04 & $(0.02)$ & $\dagger$ \\
log Visit Block $\times$ Group $\times$ Target Type & -0.02 & $(0.01)$ & $*$ \\
\hline
\end{tabular}

${ }^{\dagger} p<.1,{ }^{*} p<.05,{ }^{* *} p<.01,{ }^{* * *} p<.001 . S E$ indicates the standard error of the estimate. This regression models non-color-biased trials versus the congruent color-biased trials from Visit 1 . All factors are sum-coded: for Group, control $=-1$, synesthete $=1$; for Target Type, non-color-biased $=-1$, congruent color-biased $=1$

for synesthetes than for controls if synesthetes experience greater interference from improbable snowflake-color associations.

3. Do synesthetes maintain associations better than controls after a long-term delay? If synesthetes have superior long-term retention abilities for associations, they would correctly report snowflake-color associations with greater accuracy than controls after a long-term delay.

4. Do synesthetes experience greater interference when learning new associations? Synesthetes' RTs to congruently presented color-biased snowflakes during Visit 2 should decrease more slowly than during Visit 1 if they experience greater interference when learning new colors for the color-biased snowflakes.

We analyzed the data from the association report task administered at the beginning of Day 3 to answer the second question. The analyses answering Questions 1, 2,

Table 3 Visit 1 neutral model fixed-effect results

\begin{tabular}{lrrr}
\hline & $\beta$ & \multicolumn{2}{l}{$S E$} \\
\hline Intercept & 6.56 & $(0.05)$ & $* * *$ \\
log visit Block & -0.13 & $(0.02)$ & $* * *$ \\
Group & 0.03 & $(0.05)$ & \\
Target Type & 0.03 & $(0.02)$ & \\
log visit Block $\times$ Group & 0.04 & $(0.02)$ & $\dagger$ \\
log visit Block $\times$ Target Type & 0.01 & $(0.01)$ & $\dagger$ \\
Group $\times$ Target Type & 0.08 & $(0.02)$ & $* *$ \\
log Visit Block $\times$ Group $\times$ Target Type & 0.00 & $(0.01)$ &
\end{tabular}

${ }^{\dagger} p<.1,{ }^{* *} p<.01,{ }^{* * *} p<.001 . S E$ indicates the standard error of the estimate. Regression models non-color-biased trials versus the neutral color-biased trials from Visit 1. All factors are sum-coded: for Group, control $=-1$, synesthete $=1$; for Target Type, non-color-biased $=-1$, neutral color-biased $=1$
Table 4 Both visits congruent model fixed-effect results

\begin{tabular}{lrrrl}
\hline & \multicolumn{1}{l}{$\beta$} & \multicolumn{2}{l}{$S E$} & \\
\hline Intercept & 6.35 & $(0.04)$ & $* * *$ \\
$\log$ Visit Block & -0.14 & $(0.01)$ & $* * *$ \\
Visit & -0.11 & $(0.01)$ & $* * *$ \\
Group & 0.00 & $(0.04)$ & \\
Target Type & -0.08 & $(0.02)$ & $* *$ \\
$\log$ Visit Block $\times$ Visit & 0.02 & $(0.00)$ & $* * *$ \\
$\log$ Visit Block $\times$ Group & 0.01 & $(0.01)$ & \\
Visit $\times$ Group & 0.01 & $(0.01)$ & \\
$\log$ Visit Block $\times$ Target Type & -0.01 & $(0.00)$ & \\
Visit $\times$ Target Type & 0.02 & $(0.00)$ & $* * *$ \\
Group $\times$ Target Type & 0.03 & $(0.02)$ & \\
$\log$ Visit Block $\times$ Visit $\times$ Group & -0.01 & $(0.00)$ & $* *$ \\
$\log$ Visit Block $\times$ Visit $\times$ Target Type & 0.00 & $(0.00)$ & $\dagger$ \\
$\log$ Visit Block $\times$ Group $\times$ Target Type & -0.01 & $(0.00)$ & $*$ \\
Visit $\times$ Group $\times$ Target Type & -0.01 & $(0.00)$ & $* * * *$ \\
$\log$ Visit Block $\times$ Visit $\times$ Group $\times$ Target Type & 0.00 & $(0.00)$ & \\
\hline
\end{tabular}

${ }^{\dagger} p<.1,{ }^{*} p<.05,{ }^{* *} p<.01,{ }^{* * *} p<.001 . S E$ indicates the standard error of the estimate. This regression models non-color-biased trials versus the congruent color-biased trials from Visits 1 and 2. All factors are sum-coded: for Group, control $=-1$, synesthete $=1$; for Target Type, non-color-biased $=-$ 1 , neutral color-biased $=1$; for Visit, Visit $1=-1$, Visit $2=1$

and 4 were performed with a set of mixed-effect linear models on correct trials from the detection task (see the Data Analysis section for details).

\section{Learning of the initial pairings}

If synesthetes and nonsynesthetes learned the implicit snowflake-color associations at different rates during Visit 1, we should see group differences in their RT behavior as a function of block and target type (i.e., non-color-biased, congruent color-biased, or neutral color-biased). Specifically, synesthetes' RTs to color-biased targets presented in their congruent color should decrease more quickly than the controls'. This pattern would indicate quicker realization of the most probable color for the color-biased snowflakes.

We fit synesthetes' and controls' RT data with a mixedeffects linear regression (Visit 1 congruent model; see Table 2) to evaluate the presence of this data pattern and assess whether groups learned the shape-color associations at different rates. The results indicated that participants did indeed learn the presented associations, with average RTs to the congruent color-biased targets being faster than those to noncolor-biased targets: $\beta=-0.10, p<.001$. The facilitated RTs in response to congruent color-biased targets, however, marginally differed in magnitude across groups $(\beta=0.04, p=$ $.07)$, with synesthetes experiencing less facilitation than nonsynesthetes. Further examining RTs to the congruent 
color-biased targets, we found that synesthetes' RTs on these trials decreased more quickly than the nonsynesthetes' RTs (controlling for each group's non-color-biased RTs; see Fig. 2). A significant three-way interaction among block, group, and target type in our Visit 1 congruent model statistically confirmed this data pattern: $\beta=-0.02, p<.05$. Interestingly, it appears that this three-way interaction is driven by controls' early sensitivity to congruently presented color-biased targets. That is, nonsynesthetes exhibited faster reaction times to congruent color-biased targets from the initial block of the first visit, whereas this pattern gradually developed throughout the first visit for the synesthetes. Contrary to a general implicit-learning benefit for synesthetes, these finding suggest that synesthetes were slower to benefit from the shape-color associations in our task and benefited less from these associations.

\section{Interference during learning of the initial pairings}

Another indication of learning in our task would be slower RTs to color-biased targets when they are not presented in their congruent color. This data pattern would demonstrate an interference effect for improbable snowflake-color presentations. We evaluated whether or not synesthetes and nonsynesthetes experienced different amounts of interference while implicitly learning the snowflake-color pairings, with a mixed-effect linear regression (Visit 1 neutral; Table 3). If the groups experienced interference differently, we would expect to see that group membership would affect the difference between RTs to the non-color-biased target and RTs to the neutral color-biased targets. Our analysis revealed a significant group by target type interaction $(\beta=0.08, p<.01)$, indicating that synesthetes experienced greater interference from colorbiased targets appearing in their noncongruent colors than did nonsynesthetes. That is, synesthetes' RTs to color-biased snowflakes presented in neutral colors were slower than their RTs to the non-color-biased snowflake. As can be seen in Fig. 3, controls' RTs to neutral color-biased targets are numerically faster than their RTs to the non-color-biased snowflakes. These results demonstrate that synesthetes experienced greater interference for improbable snowflake-color pairs than did controls during the learning of these shape-color associations.

\section{Memory for learned associations}

To determine whether synesthetes' and nonsynesthetes' long-term retentions of implicitly learned associations differed, we analyzed performance on the association report task obtained at the beginning of Day 3. Considering only the color-biased target snowflakes, we calculated accuracy in a binary manner: correct if participants reported an association and selected the correct color from the six options, incorrect if they chose the wrong color or did not report an association. To assess long-term memory of learned associations fairly across groups, we considered only the participants who had correctly reported both snowflake-color pairings at the end of Day 2 (four controls, seven synesthetes). Of the four controls who had demonstrated successful learning of both associations at the end of Day 2, one correctly reported both pairings during the delayed retest, and the remaining three reported neither association correctly (see Fig. 4). In contrast, five synesthetes demonstrated long-term memory of both associations, one

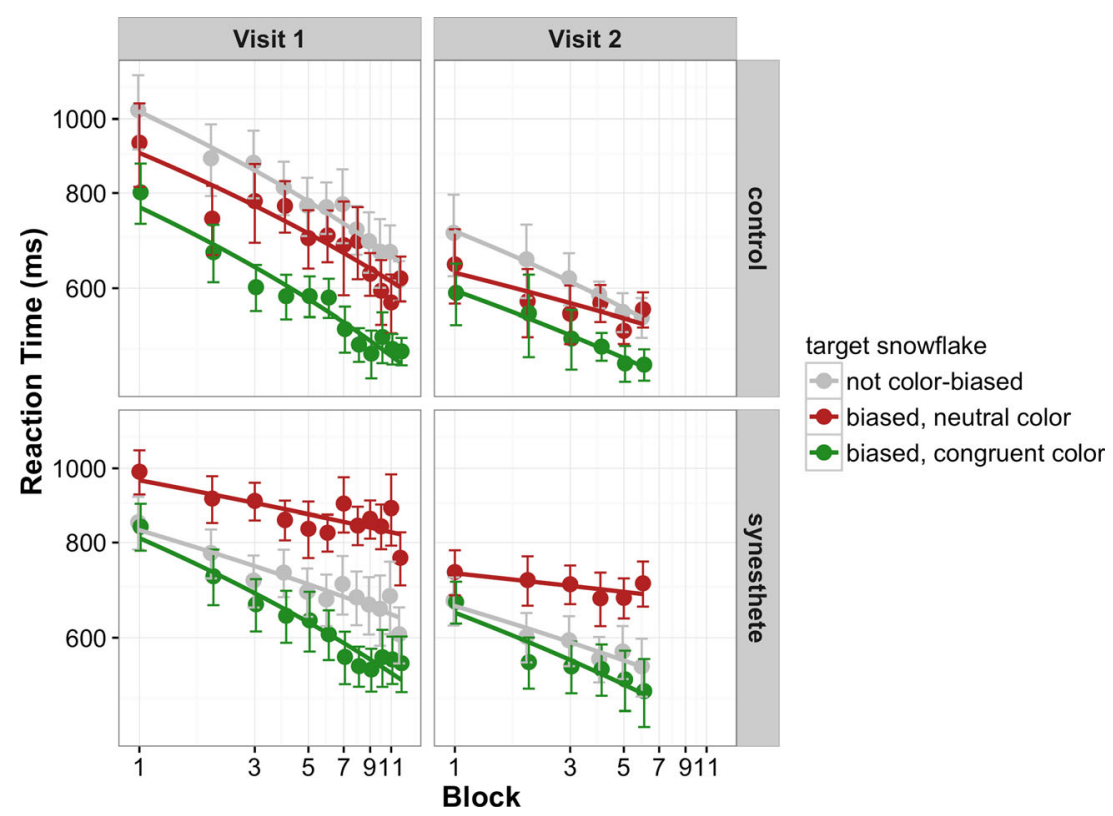

Fig. 2 Detection task RTs by block for each visit, group, and target snowflake type. Lines depict linear model fits for illustration purposes only. Error bars indicate standard errors of the means 


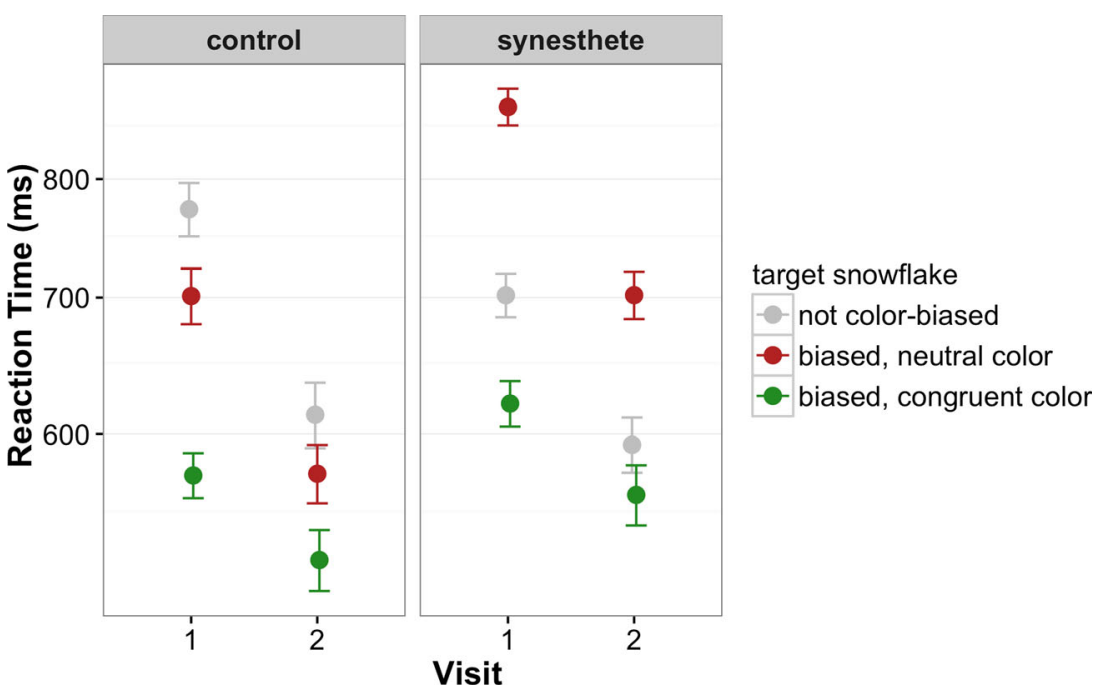

Fig. 3 Mean detection task RTs by visit, group, and target snowflake type. Error bars indicate standard errors of the means

synesthete correctly reported one association, and only one synesthete failed to remember either association. This pattern of results suggests that synesthetes have heightened long-term memory abilities for associations. We statistically confirmed this observation with a mixed-effects logistic regression modeling accuracy by group and random intercepts by participant. We found that group membership significantly predicted the long-term retention of snowflake-color associations, with controls having lower average accuracy than synesthetes: $\beta=-2.40, p<.05$. These findings suggest that synesthetes have a heightened ability to retain associations over long periods of time.

\section{Interference when learning new pairings}

Finally, we investigated whether or not synesthetes experience greater interference when learning new associations.

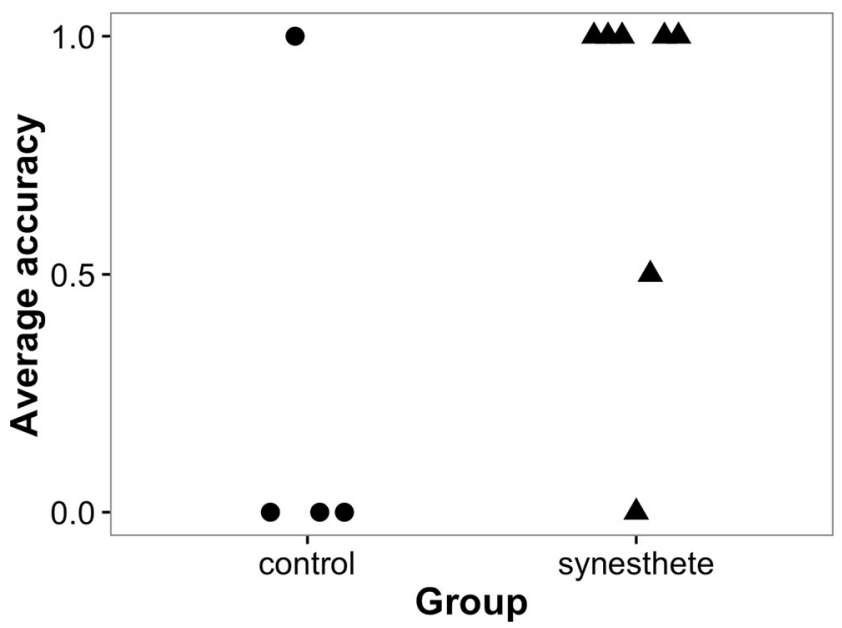

Fig. 4 Long-term retention of snowflake-color associations. Each point represents one participant. Only those participants who correctly reported both associations at the end of Day 2 are included
These new associations paired the same color-biased target snowflakes from Visit 1 with colors that had previously been neutral. We hypothesized that if interference were experienced while learning novel associations, the difference between the RTs to newly congruent color-biased targets and to non-colorbiased targets would be smaller during Visit 2 than during Visit 1 . That is, the RT benefit would be smaller for seeing the color-biased snowflakes in their newly assigned most probable color. If the groups differed in the amounts of interference experienced while learning the new associations, we should find that group membership would interact with target type and visit. The results from a mixed-effects linear model (both visits congruent; see Table 4 for details) indicated a significant three-way interaction among group, target type, and visit; $\beta=-0.01, p<.001$. As can be seen in Fig. 2, this finding indicates that the synesthetes' congruent facilitation that had been evident during Visit 1 disappeared during the second visit, whereas controls exhibited a consistent benefit from seeing a target snowflake in its congruent color during both visits. That is, synesthetes did not respond faster to congruently presented color-biased targets during Visit 2 . These results suggest that synesthetes' heightened long-term memory for shape-color pairs leads to increased interference when learning new colors for those same shapes.

\section{Generalized group differences}

We also compared group performance on the Corsi blocktapping task to ensure that our synesthetes did not have superior memory in general or increased motivation. A $t$ test did not reveal significant memory span differences between the controls and synesthetes, $M_{C}=5.83, M_{S}=5.29, t(13)=-1.28$, $p>.05$. This result suggests that our synesthetes and nonsynesthetes differed neither in general working memory abilities nor in motivation put forth during our experiment. 
Moreover, since working memory is highly correlated with intelligence, this finding also suggests that the groups did not differ in general intelligence (e.g., Conway, Kane, \& Engle, 2003).

\section{Discussion}

In the present study, we investigated the hypothesis that synesthesia arises in part due to an enhanced ability to implicitly learn associations that are present in the statistics of the real world. Mimicking the environment in which synesthesia is acquired, we probabilistically paired snowflakes with colors, and participants were not required to attend to these colors to complete the detection task. We collected a continuous accuracy measure at multiple time points across learning, allowing for fine-grained investigation of associative implicit learning in synesthetes and nonsynesthetes.

Our results regarding learning of the initial snowflake-color pairs revealed strikingly different patterns of learning for synesthetes and nonsynesthetes. Both synesthetes and nonsynesthetes were able to implicitly learn the snowflakecolor associations, but the learning patterned in different ways for each group. Nonsynesthetes exhibited a fast, strong facilitation effect for color-biased snowflakes presented in their congruent colors. In contrast, synesthetes slowly acquired a reduced version of this RT facilitation effect over the course of Visit 1. We also reported group differences in RTs to colorbiased snowflakes occurring in neutral colors. For these trials, synesthetes' RTs were slower than those to non-color-biased targets, whereas nonsynesthetes did not experience such an interference effect. This pattern of results indicates that synesthetes experienced greater interference when colorbiased targets were presented in a color other than their congruent color. Overall, our findings from Visit 1 demonstrate a "fast-facilitation" learning effect for controls, and a "fast-interference, slow-facilitation" learning effect for synesthetes.

Further group differences were found when addressing long-term retention of the original snowflake-color associations and learning of novel associations. After a two-week delay, synesthetes demonstrated superior retention of the original associations, relative to nonsynesthetes. Potentially as a consequence of this superior long-term memory, synesthetes also experienced greater interference than did nonsynesthetes when learning new associations. Specifically, synesthetes did not exhibit a facilitation effect for color-biased targets presented in their newly congruent colors. By testing participants after a two-week delay on the original associations and then teaching them novel association, we found that synesthetes had superior long-term memory and, consequently, experienced greater interference when learning new colors for the same color-biased snowflakes.
This study demonstrated that learning implicit shapecolor pairs affects synesthetes and nonsynesthetes in different ways. We expanded upon findings from the single previous study that had investigated implicit learning in synesthesia. Rothen et al. (2013) presented participants with artificial grammars and only found implicit-learning differences for stimuli within the synesthetes' synesthetic domain (i.e., graphemes for grapheme-color synesthetes). Our findings add further detail to the ways in which synesthetes' implicit learning differs from nonsynesthetes', in a novel domain: associative learning. The pattern of our results also partially supports our recent study of explicit associative learning in synesthetes, in which we found superior long-term memory but a faster learning rate of associations for synesthetes (Bankieris \& Aslin, in press). There are a few potential explanations for the contradictory findings for learning rates in explicit versus implicit tasks. Since the explicit-learning task we used was self-directed and the present implicit-learning task was experimenter-directed, this difference could contribute to the conflicting results. Perhaps explicitly choosing which items to allocate their attention to allows synesthetes to more efficiently consolidate what they learn, whereas experimenterdefined presentation sequences prevent this enhanced consolidation. Although we did not find evidence for group differences in self-directed learning strategies, the findings from previous studies investigating explicit associative learning support the hypothesis that self-directed learning is a critical factor to consider. Multiple studies using experimenter-directed presentation of explicitly learned associations either have failed to find an enhanced learning rate or have actually demonstrated a reduced learning rate for synesthetes (Brang, Ghiam, \& Ramachandran, 2013; Pfeifer, Rothen, Ward, Chan, \& Sigala, 2014; Pritchard, Rothen, Coolbear, \& Ward, 2013). The one other study using a self-directed learning phase had presented participants with six shape-color associations simultaneously, allowing the participants to direct their attention to individual pairings as desired (Rothen \& Meier, 2010). That study did not directly investigate learning rates, but it showed that synesthetes outperformed controls on both an immediate and a delayed test. Accordingly, thoughtfully considering the learning phase in future research will be necessary in order to gain a deeper understanding of synesthetes' associative memory capabilities.

Another potential explanation for the different learning rate results between explicit and implicit studies is the type and granularity of memory that was assessed at test. For the explicit-learning and-memory study we conducted (Bankieris \& Aslin, in press), participants were required to recall the precise color values of the learned associations. In 
contrast, in the present study investigating implicit learning and memory, we essentially tested recognition memory of various statistics. Our explicit study also tested knowledge of associations at a much finer granularity than in this implicit study. Accordingly, these are yet two more variables that should be considered when designing future studies.

One final potential reason that group learning rate differences were observed in our implicit versus our explicit task might be the reliability of the statistics presented in these tasks. In the explicit association task, shapes appeared in one color $100 \%$ of the time, whereas the statistics in the implicit task were probabilistic. Since we have demonstrated that synesthetes are more affected by improbable pairings or statistical occurrences, it could be that this interference causes synesthetes to be more negatively affected by the "noise" of probabilistic statistics, and therefore to learn them more slowly than controls. To test this possibility, studies requiring synesthetes to explicitly learn probabilistic associations should be carried out. Overall, our research suggests that synesthetes learn associations in a different manner than do nonsynesthetes and have an enhanced ability to retain associations for long periods of time.

\section{References}

Asher, J. E., Lamb, J. A., Brocklebank, D., Cazier, J.-B., Maestrini, E., Addis, L., ... Monaco, A. P. (2009). A whole-genome scan and finemapping linkage study of auditory-visual synesthesia reveals evidence of linkage to chromosomes $2 \mathrm{q} 24,5 \mathrm{q} 33,6 \mathrm{p} 12$, and $12 \mathrm{p} 12$. American Journal of Human Genetics, 84, 279-285.

Bankieris, K., \& Aslin, R. (in press). Explicit associative learning in synesthetes and nonsynesthetes. i-Perception.

Bankieris, K., \& Simner, J. (2014). Sound symbolism in synesthesia: Evidence from a lexical-gustatory synesthete. Neurocase, 20, 640-651. doi:10.1080/13554794.2013.826693

Brang, D., Ghiam, M., \& Ramachandran, V. S. (2013). Impaired acquisition of novel grapheme-color correspondences in synesthesia. Frontiers in Human Neuroscience, 7, 717. doi:10.3389/fnhum.2013.00717

Calkins, M. W. (1893). A statistical study of pseudochromesthesia and of mental forms. American Journal of Psychology, 5, 439-466.

Claparede, E. (1903). Persistance de l'audition colorée. Comptes Rendus des Seances de la Societe de Biologie et de ses Filiales, 55, 1257-1259.

Conway, A. R. A., Kane, M. J., \& Engle, R. W. (2003). Working memory capacity and its relation to general intelligence. Trends in Cognitive Sciences, 7, 547-552. doi:10.1016/j.tics.2003.10.005
Eagleman, D. M., Kagan, A. D., Nelson, S. S., Sagaram, D., \& Sarma, A. K. (2007). A standardized test battery for the study of synesthesia. Journal of Neuroscience Methods, 159, 139-145. doi:10.1016/j. jneumeth.2006.07.012

Grossenbacher, P. G., \& Lovelace, C. T. (2001). Mechanisms of synesthesia: Cognitive and physiological constraints. Trends in Cognitive Sciences, 5, 36-41. doi:10.1016/S1364-6613(00)01571-0

Kusnir, F., \& Thut, G. (2012). Formation of automatic letter-colour associations in non-synaesthetes through likelihood manipulation of letter-colour pairings. Neuropsychologia, 50(14), $3641-3652$.

Marks, L. E. (1974). On associations of light and sound: The mediation of brightness, pitch, and loudness. American Journal of Psychology, 87, 173-188.

Marks, L. E. (1987). On cross-modal similarity: Auditory-visual interactions in speeded discrimination. Journal of Experimental Psychology: Human Perception and Performance, 13, 384-394. doi:10.1037/0096-1523.13.3.384

Mueller, S. T. (2011). The PEBL Corsi Block Test. Computer software retrieved from $\mathrm{http}: / /$ pebl.sf.net

Pfeifer, G., Rothen, N., Ward, J., Chan, D., \& Sigala, N. (2014). Associative memory advantage in grapheme-color synesthetes compared to older, but not young adults. Frontiers in Psychology, 5, 696. doi:10.3389/fpsyg.2014.00696

Pritchard, J., Rothen, N., Coolbear, D., \& Ward, J. (2013). Enhanced associative memory for colour (but not shape or location) in synaesthesia. Cognition, 127, 230-234. doi:10.1016/j.cognition.2012.12.012

Ramachandran, V., \& Hubbard, E. M. (2001). Synaesthesia: A window into perception, thought and language. Journal of Consciousness Studies, 8, 3-34.

Rothen, N., \& Meier, B. (2010). Higher prevalence of synaesthesia in art students. Perception, 39, 718-720. doi:10.1068/p6680

Rothen, N., Scott, R. B., Mealor, A. D., Coolbear, D. J., Burckhardt, V., \& Ward, J. (2013). Synesthetic experiences enhance unconscious learning. Cognitive Neuroscience, 4, 231-238.

Simner, J., \& Ludwig, V. U. (2012). The color of touch: A case of tactilevisual synaesthesia. Neurocase, 18, 167-180.

Simner, J., Ward, J., Lanz, M., Jansari, A., Noonan, K., Glover, L., \& Oakley, D. (2005). Non-random associations of graphemes to colours in synaesthetic and non-synaesthetic populations. Cognitive Neuropsychology, 22, 1069-1085.

Smilek, D., Carriere, J. S. A., Dixon, M. J., \& Merikle, P. M. (2007). Grapheme frequency and color luminance in grapheme-color synaesthesia. Psychological Science, 18, 793-795. doi:10.1111/j.14679280.2007.01981.x

Ward, J., Huckstep, B., \& Tsakanikos, E. (2006). Sound-colour synaesthesia: To what extent does it use cross-modal mechanisms common to us all? Cortex, 42, 264-280.

Witthoft, N., \& Winawer, J. (2013). Learning, memory, and synesthesia. Psychological Science, 24, 258-265. doi:10.1177/0956797612452573

Witthoft, N., Winawer, J., \& Eagleman, D. M. (2015). Prevalence of learned grapheme-color pairings in a large online sample of synesthetes. PLOS ONE, 10, e0118996. doi:10.1371/journal. pone. 0118996 